\title{
叶片式气液混输洜全流道内流场特性分析 ${ }^{*}$
}

\author{
张文武 余志毅 李泳江 程学良 \\ (北京理工大学机械与车辆学院 北京 100081)
}

\begin{abstract}
摘要: 有关叶片式气液混输葲全流道内流场特性的研究还不充分, 因此选取空气-水作为输运介质, 基于 ANSYS_CFX 对一 叶片式气液混输百进行全流道数值模拟。计算域采用 ICEM_CFD 和 TurboGrid 进行了结构化网格划分。通过数值计算获取的 外特性数据与试验数据进行对比, 数值计算方法的可靠性得到了验证。计算结果显示, 不同进口含气率下叶轮流道内的气体 主要聚集在叶轮出口轮毫处的吸力面附近且随着进口含气率的增加, 气体在该处的聚集程度增强, 分布的不均匀度增加。9\%、 $15 \%$ 和 $21 \%$ 进口含气率下叶轮内流体的最大湍动能分别是 $3 \%$ 进口含气率下的 1.07 倍、 1.53 倍和 1.83 倍。不同进口含气率下 导叶内的气体均在轮㪍处聚集, 且沿着流动方向, 轮毫处的气体逐渐向主流区扩散。9\%进口含气率下, 叶轮内气体的聚集程 度随着流量的增加逐渐减小, 而导叶内的气体在设计流量 $\left(Q_{\mathrm{d}}\right)$ 时聚集程度最大, 大流量 $\left(1.25 Q_{\mathrm{d}}\right)$ 次之, 小流量 $\left(0.75 Q_{\mathrm{d}}\right)$ 最小。 以上研究结果更深入地揭示了叶片式气液混输葲的内流场特性, 可以为该类洜的优化设计提供参考, 提高其输运效率。
\end{abstract} 关键词: 进口含气率; 气液混输原; 流场特性; 旋浴; 数值模拟 中图分类号: TH312

\section{Flow Characteristics Analysis for the Whole Flow Passage of a Multiphase Rotodynamic Pump}

\author{
ZHANG Wenwu YU Zhiyi LI Yongjiang CHENG Xueliang
}

(School of Mechanical Engineering, Beijing Institute of Technology, Beijing 100081)

\begin{abstract}
Research on the flow characteristics for the whole flow passage of a multiphase rotodynamic pump is insufficient. Therefore, based on ANSYS_CFX, simulations are performed for the flow in such a pump with the medium of air-water. Structured grids for the computational domain are generated using ICEM_CFD and TurboGrid. The reliability of simulation is verified by comparing with the experimental data. The results show that, under different inlet gas void fraction (IGVF) conditions, the gas in the impeller mainly accumulated at the impeller hub and the closer to the impeller outlet and the blade suction surface, the higher degree for gas aggregation. With the increase of IGVF, the degree of gas aggregation in the impeller, as well as the gas inhomogeneity, is higher. The maximum value of the turbulence kinetic energy at IGVF=9\%, 15\% and $21 \%$ are respectively $1.07,1.53$ and 1.83 times than that at $\mathrm{IGVF}=3 \%$. Meanwhile, the gas in the diffuser mainly accumulated near the hub and gradually spread toward the mainstream region along the flow direction. For $I G V F=9 \%$, the degree of gas aggregation in the impeller decreased gradually with the increase of flow rate, while in the diffuser, it is the largest at design condition $\left(Q_{\mathrm{d}}\right)$, followed by the large flow rate condition $\left(1.25 Q_{\mathrm{d}}\right)$, then the small flow rate condition $\left(0.75 Q_{\mathrm{d}}\right)$. The results above give a more in-depth understanding about the flow characteristics in such pumps, which will help in the optimization design and obtaining ahigher pump efficiency.
\end{abstract}

Key words: inlet gas void fraction (IGVF); gas-liquid multiphase pump; flow characteristics; vortex; numerical simulation

\section{0 前言}

随着科技的发展和工业上对气液混合输运的需 求, 气液混输洜不仅被广泛地应用于石油工业, 而 且在化工、食品、城市给水以及核工业等领域也有 广泛的应用 ${ }^{[1-3]}$ 。用于气液混输的泵型通常有两种,

* 国家自然科学基金(51579006)和北京理工大学基础研究基金(20150342012) 资助项目。20180522 收到初稿, 20181030 收到修改稿
一种是容积百, 一种是叶片泵。叶片泵与容积洜相 比, 它具有体积小, 输送流量大, 制造精度要求不 高, 对流场中固体颗粒的敏感性低，易于使用和维 修等优点 ${ }^{[4]}$ 。

近年来, 气液混输洜的研究主要集中在优化设 计和混输特性等方面。如优化设计方面: KIM 等 ${ }^{[5]}$ 通过试验设计技术和数值分析方法对一用响应面方 法设计的混输洜进行了优化, 提高了其性能。CAO 等 ${ }^{[6]}$ 结合反问题和 CFD 分析方法设计了一叶片式混 
输葲的叶轮, 并试验证实了该混输葲具有流量范围 宽、效率高的特点。混输特性方面: MURAKAMI 等 ${ }^{[7-10]}$ 均通过高速摄影技术对泵内的流型进行了研 究, 将其划分为孤立泡状流、泡状流、气囊流和分 离气体流。CARIDAD 等 ${ }^{[11]}$ 在气液两相工况下对一 离心泵进行了数值模拟, 发现洜的扬程随进口气泡 直径的增大而降低。综上所述, 学者虽然对混输泵 的设计方法进行了各种探索, 并做了大量的试验研 究工作, 但由于试验具有周期长, 花费高等缺点, 且根据当前的监测技术, 有些参数是无法通过试验 获取的。因此, CFD 仿真技术逐渐成为研究混输百 性能及内部流动特性的一种重要手段 ${ }^{[12]}$ 。事实上, 由于叶轮的旋转和动静干涉作用, 叶片泵内往往伴 有气泡的聚合与分裂、两相的分离和混合等复杂现 象 $^{[13]}$, 学者针对叶片式气液混输泵内流场特性的研 究还不是很充分。

本研究选取空气-水作为输运介质, 对一叶片式 气液两相混输泵内的流动进行了全流道三维数值模 拟, 对不同进口含气率和不同流量下叶轮和导叶流 道内的含气率分布及内流场特性进行了探讨, 以对 叶片式气液混输泵的内部流动特性有进一步的理 解, 从而为该类泵的优化设计提供参考, 进而提高 泵的输运效率。

\section{1 试验系统}

\section{1 试验原理}

图 1 是该叶片式气液混输泵的试验系统原理 图, 考虑到试验的安全性及设备的可重复性使用, 试验介质选取的是空气和水。试验过程中, 由压缩 机提供的空气, 经减压阀调节压力后与水在均混器 中均匀混合, 然后一起进入混输洜, 经过原的做功 作用, 二者一起流回水箱。在试验系统中, 气体管

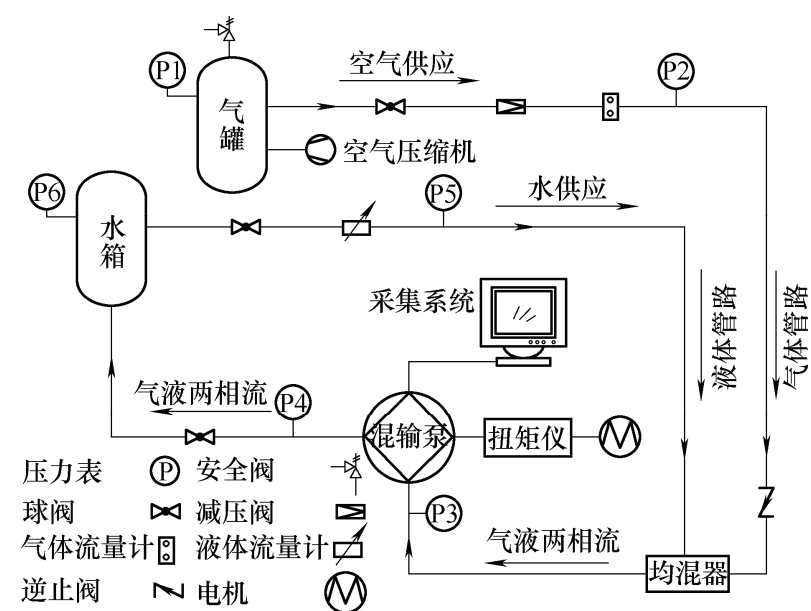

图 1 试验系统原理图
路和液体管路的流量分别由浴轮流量计和转子流量 计测量(精度等级均为 $\pm 1.5 \%$ ); 泵转速、功率和扭矩由 一型号为 CYT-302 的扭矩功率仪测量 (精度为 $\pm 0.2 \%$ )。

\section{2 模型及参数}

图 2.3 分别是该叶片式气液混输洜的试验模型 和数值计算模型, 包括进口管、叶轮、导叶和出口 管四部分。该百的设计转速为 $2950 \mathrm{r} / \mathrm{min}$, 设计流 量为 $50 \mathrm{~m}^{3} / \mathrm{h}$, 设计扬程为 $15 \mathrm{~m}$, 叶轮和导叶的主要 设计参数尺寸如表 1 所示。同时, 为了便于用高速 摄影机观察洜内的气液两相流动状态, 试验洜模型 的叶轮和导叶外壳均用有机玻璃加工而成。图 4 是 用高速摄影机拍摄的 $9 \%$ 进口含气率下的洜内流场。 由图 4 可知, 叶轮流道内的气体主要在轮勃处聚集, 且在叶片吸力面聚集的强度大于压力面。

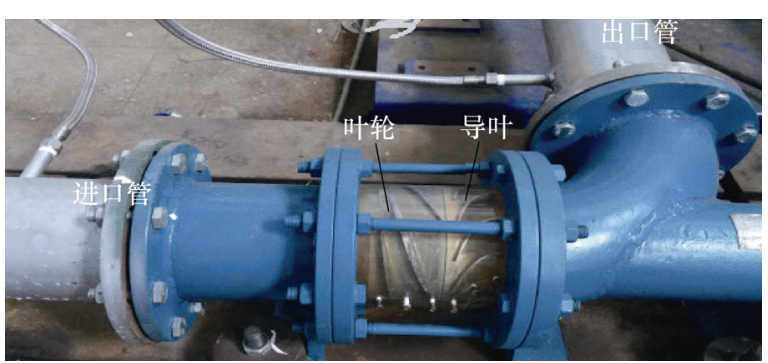

图 2 试验葲模型

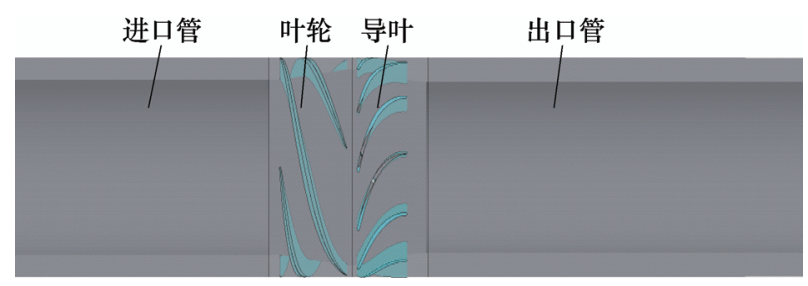

图 3 数值计算模型

表 1 混输泵叶轮和导叶主要设计参数

\begin{tabular}{lcc}
\hline \multicolumn{1}{c}{ 参数 } & 叶轮 & 导叶 \\
\hline 轮缘直径 $D_{\mathrm{s}} / \mathrm{mm}$ & 150 & 150 \\
进口轮毂直径 $D_{\mathrm{h} 1} / \mathrm{mm}$ & 120 & 134 \\
出口轮毂直径 $D_{\mathrm{h} 2} / \mathrm{mm}$ & 134 & 120 \\
轴向长度 $b / \mathrm{mm}$ & 60 & 40 \\
叶片数 $z$ & 4 & 11 \\
\hline
\end{tabular}

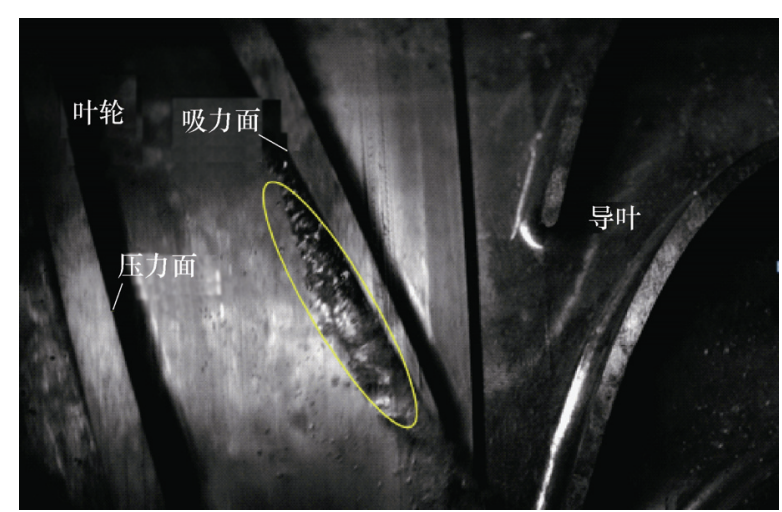

图 4 9\%进口含气率下试验葲内流场 


\section{2 数值计算方法}

\section{1 控制方程}

本研究选取双流体模型作为该气液混输洜内流 动预测的两相流模型 ${ }^{[14-17]}$, 在该模型中, 每种介质 均建立一组守恒方程, 即它们有各自的速度场, 但 共用压力场，考虑了介质间的相互作用，因此可以 满足本研究 3\% 21\%进口含气率范围的要求 ${ }^{[18-19]}$ 。 同时, 基于 ANSYS_CFX 16.0 对定常的雷诺平均 $\mathrm{N}-\mathrm{S}$ 方程进行求解。在笛卡儿坐标系中, 不可压缩 流体的控制方程如下 ${ }^{[20-23]}$ 。

连续方程

$$
\nabla \cdot\left(\alpha_{k} \rho_{k} \boldsymbol{U}_{k}\right)=0
$$

动量方程

$$
\nabla \cdot\left(\alpha_{k} \rho_{k} \boldsymbol{U}_{k} \boldsymbol{U}_{k}-\alpha_{k} \boldsymbol{\tau}_{k}\right)=-\alpha_{k} \nabla p+\boldsymbol{M}_{k}+\alpha_{k} \rho_{k} f_{k}
$$

式中, 下标 $k=1$ 或 $\mathrm{g}$ 分别表示液相或气相; $\rho_{k}$ 为 密度; $\alpha_{k}$ 为体积分数且 $\alpha_{1}+\alpha_{\mathrm{g}}=1 ; p$ 为压力; $\boldsymbol{U}_{k}$ 为速度; $\boldsymbol{M}_{k}$ 为相间作用力 $; \boldsymbol{f}_{k}$ 为质量力 $; \boldsymbol{\tau}$ 是关于 流体黏度和湍动黏度的黏性应力张量, 它的分量 可写为

$$
\tau_{k, i j}=2 \mu_{k} s_{i j}-\rho_{k} \overline{w_{k i}^{\prime} w_{k j}^{\prime}}
$$

式中, $i, j=1,2,3$ 表示叶轮旋转相对坐标系的三个 方向; $\mu_{k}$ 动力黏度; $s_{i j}$ 为应力张量; $\rho_{k} w_{k i}^{\prime} w_{k j}^{\prime}$ 表示 由于湍流运动而引起的湍流雷诺应力, 表达式如下

$$
-\rho_{k} \overline{w_{k i}^{\prime} w_{k j}^{\prime}}=2 \mu_{\mathrm{t}} s_{i j}-\frac{2}{3} \rho_{k} k \delta_{i j}
$$

式中, $\mu_{\mathrm{t}}$ 的求解采用基于 $k-\omega$ 的剪切应力输运模型, 该模型结合了 $k-\varepsilon$ 和 $k-\omega$ 模型的模化思想, 近壁面 采用 $k-\omega$ 模型, 主流区采用 $k-\varepsilon$ 模型 ${ }^{[24]} 。 \mu_{\mathrm{t}}$ 的计算 式如下

$$
\mu_{\mathrm{t}}=\frac{\rho_{\text {mix }} a_{1} k}{\max \left(a_{1} \omega, S F_{2}\right)}
$$

式中, $a_{1}$ 为模型常数 $\left(a_{1}=5 / 9\right) ; \rho_{\text {mix }}$ 为气液混合密 度; $S$ 为应变率的不变测度; $F_{2}$ 是混合函数; $k$ 和 $\omega$ 分别为湍动能和湍流频率。

\section{2 计算域网格}

计算域进出口管、叶轮和导叶四部分的整体网 格及叶轮和导叶前缘局部放大网格如图 5 所示, 其 中进出口管采用 ICEM_CFD16.0 进行结构网格划 分, 为了提高网格质量, 采用 TurboGrid16.0 对叶轮 和导叶分别建立了 $\mathrm{H} / \mathrm{J} / \mathrm{C} / \mathrm{O}$ 型等拓扑结构, 并进行 了结构网格划分。

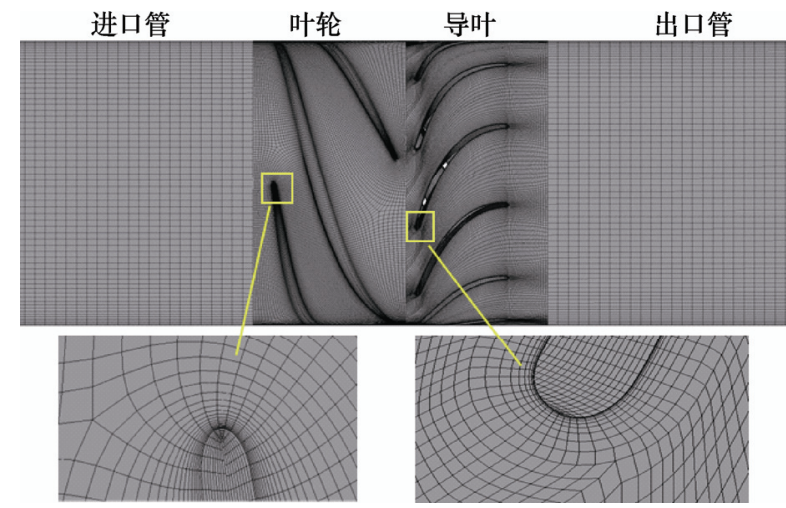

图 5 计算域网格

为了能够充分利用计算资源, 减小计算机存储 及耗时等问题，在纯水设计工况下进行了网格无关 性分析, 如表 2 所示。由表 2 可知, III 和 IV 两种 网格密度下的扬程和效率相差较小, 因此, 最终选 取第 III 种网格进行计算分析。

表 2 网格无关性分析

\begin{tabular}{ccccc}
\hline & I & II & III & IV \\
\hline 进口管 & 54516 & 54516 & 54516 & 54516 \\
叶轮 & $165096 \times 4$ & $306660 \times 4$ & $590416 \times 4$ & $809361 \times 4$ \\
导叶 & $35168 \times 11$ & $56280 \times 11$ & $105840 \times 11$ & $129472 \times 11$ \\
出口管 & 99540 & 99540 & 99540 & 99540 \\
总网格数 $N /$ 百万 & 1.20 & 2.00 & 3.68 & 4.82 \\
扬程 $H / \mathrm{m}$ & 17.79 & 17.55 & 17.24 & 17.23 \\
扬程误差 $\Delta H(\%)$ & 6.24 & 4.82 & 2.97 & 2.88 \\
效率 $\eta(\%)$ & 59.16 & 58.73 & 58.04 & 57.99 \\
效率误差 $\Delta \eta(\%)$ & 2.89 & 2.14 & 0.94 & 0.84 \\
\hline
\end{tabular}

\section{3 边界条件及数值求解}

计算时, 参考压力设置为 1 个标准大气压, 进 口给定空气和水的总质量流量、体积分数及中等湍 流强度；基于混合介质属性的计算方法 ${ }^{[6]}$ ，根据泵 进口设计参数，可计算出 $3 \% 、 9 \% 、 15 \%$ 和 $21 \%$ 进 口含气率下混合介质的密度、动力黏度和雷诺数, 如表 3 所示。出口给定平均压力, 相对压力设置为 0 ; 所有壁面对两相均采用速度无滑移条件; 动静交 界面(进口管-叶轮交界面、叶轮-导叶交界面)采用冻 结转子法, 该方法能对多重参考系问题进行稳定的 求解。本研究采用定常数值求解, 对流项和湍动能 相均采用二阶迎风格式求解，计算迭代步数设置为 2000 步, 收敛残差 RMS 为 $1 \times 10^{-4}$ 。

表 3 计算域进口空气和水的雷诺数

\begin{tabular}{cccc}
\hline 进口含气率 $(\%)$ & $\begin{array}{c}\text { 密度 } \\
\rho /\left(\mathrm{kg} \cdot \mathrm{m}^{-3}\right)\end{array}$ & $\begin{array}{c}\text { 动力䇿度 } \\
\mu /(\mathrm{mPa} \cdot \mathrm{s})\end{array}$ & $\begin{array}{c}\text { 雷诺数 } \\
\operatorname{Re}\end{array}$ \\
\hline 3 & 967.13 & 0.93 & 68248.69 \\
9 & 907.38 & 0.99 & 59833.76 \\
15 & 847.63 & 1.04 & 53260.22 \\
21 & 787.88 & 1.08 & 47982.18 \\
\hline
\end{tabular}




\section{3 结果与讨论}

\section{1 数值结果与试验结果对比}

图 6 是转速为 $2950 \mathrm{r} / \mathrm{min}$, 纯水工况不同流量 下的数值计算结果与试验结果对比。由图 6 可知, 计算获取的效率和扬程特性曲线与试验曲线吻合良 好, 其中设计流量下效率和扬程的相对误差分别为 $0.94 \%$ 和 $2.97 \%$ 。表 4 是气液总流量为 $50 \mathrm{~m}^{3} / \mathrm{h}$, 不 同含气率下的数值计算扬程与试验扬程对比。由表 4 可知, 转速为 $2950 \mathrm{r} / \mathrm{min}$, 进口含气率 $\mathrm{IGVF}=9 \%$, $15 \%$ 和 $21 \%$ 下数值扬程与试验扬程相差较小，相对 误差分别为 $1.89 \% 、 3.27 \%$ 和 $1.45 \%$ 。以上分析说明 了该研究所采用的数值计算方法是可靠的。

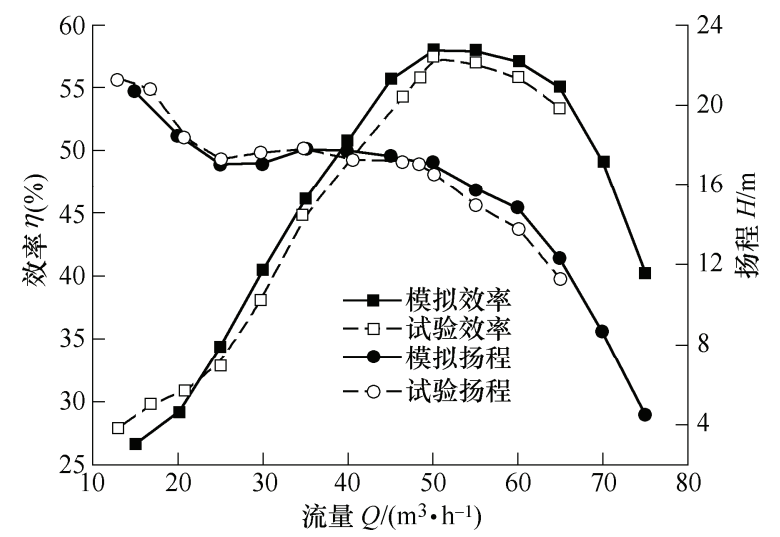

图 6 纯水工况下泵的外特性(效率和扬程)对比

表 4 不同进口含气率下数值计算结果和试验结果对比

\begin{tabular}{cccc}
\hline 工况 & $\begin{array}{c}\text { 计算泉扬程 } \\
H_{-} \mathrm{CFD} / \mathrm{m}\end{array}$ & $\begin{array}{c}\text { 试验㫤扬程 } \\
H_{-} \mathrm{EXP} / \mathrm{m}\end{array}$ & 误差 $\Delta(\%)$ \\
\hline $\mathrm{IGVF}=9 \%$ & 16.19 & 15.89 & 1.89 \\
$\mathrm{IGVF}=15 \%$ & 15.45 & 14.96 & 3.27 \\
$\mathrm{IGVF}=21 \%$ & 14.71 & 14.50 & 1.45 \\
\hline
\end{tabular}

\section{2 进口含气率对叶轮流道内流场特性的影响}

图 7 是不同进口含气率 $(\mathrm{IGVF}=3 \%, 9 \%, 15 \%$, $21 \%$ )下叶轮进口到出口轴面的平均含气率分布(面 积平均)。由图 7 可知, 不同进口含气率下叶轮流道 内气体主要在轮㪘处聚集, 这是因为由于叶轮的旋 转效应, 液相的密度较大, 受到的离心力大于气相, 使液体向轮缘处移动, 气体向轮㲄处移动。同时, 随着进口含气率的增加, 气体在叶轮流道轮晸处的 聚集程度增强, 且气体分布的不均匀度增加, 从而 使葲的流动状态更加紊乱, 这也是表 4 中随着进口 含气率的增加, 泵扬程下降的原因。

图 8 是各进口含气率下叶轮轮毂 $1 / 10$ 叶高处的 平均含气率分布。由图 8 可知, 不同进口含气率下 叶轮出口轮毂处的气体主要聚集在叶片的吸力面附

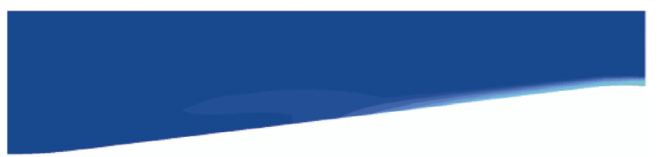

(a) $\mathrm{IGVF}=3 \%$

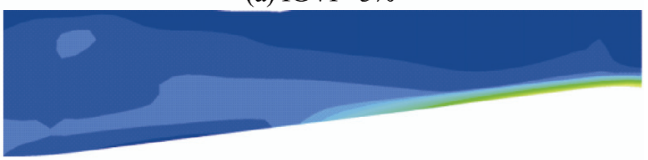

(b) $\mathrm{IGVF}=9 \%$

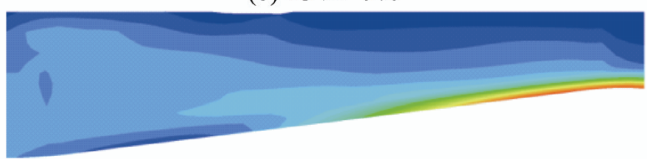

(c) $\mathrm{IGVF}=15 \%$

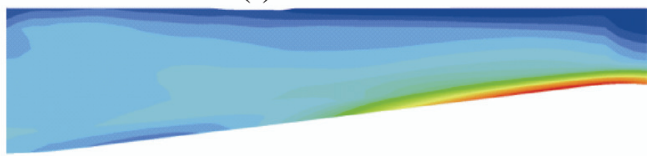

(d) $\mathrm{IGVF}=21 \%$

图 7 不同进口含气率下叶轮流道轴面平均含气率分布

近, 且随着进口含气率的增加, 气体在吸力面的聚 集程度增大。同时, 由图 8 还可知, 叶轮四个流道 内的气体分布是不对称的, 说明了混输百内气液两 相流动状态比较紊乱, 随着含气率的增加, 部分流 道可能会先发生 “气堵” 现象。

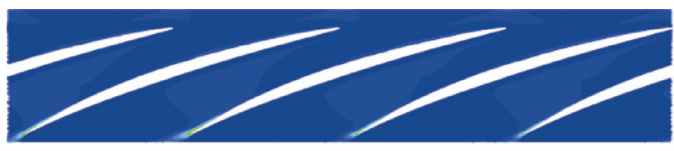

(a) $\mathrm{IGVF}=3 \%$

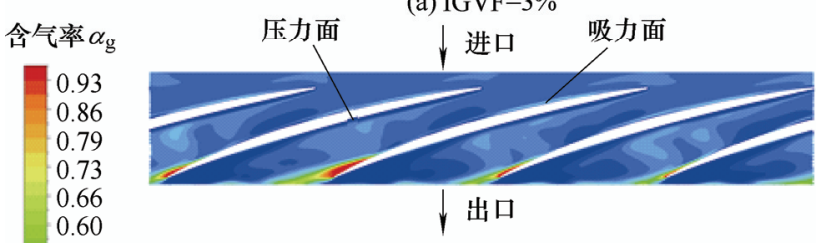

(b) $\mathrm{IGVF}=9 \%$

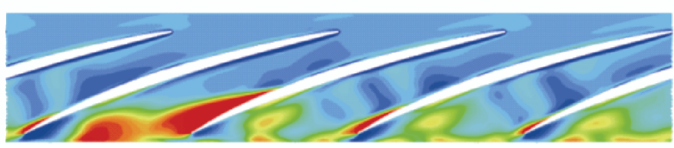

(c) $\mathrm{IGVF}=15 \%$

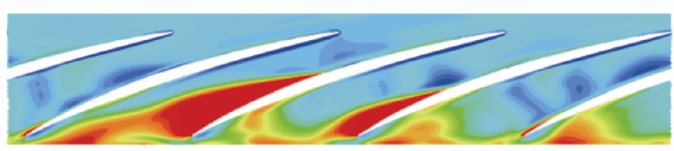

(d) $\mathrm{IGVF}=21 \%$

图 8 不同进口含气率下叶轮轮毂处含气率分布

由以上分析可知, 不同进口含气率下, 叶轮流 道内的气体主要在叶轮出口轮瑴处的吸力面聚集 (与图 4 试验结果一致)。这一方面是因为由于叶轮 的做功作用, 气体向出口方向移动的同时受到叶片 正背面压差的作用, 进而向叶片吸力面移动; 另一 方面是因为沿着流动方向, 叶轮流道内的压力逐渐 增大; 同时, 叶轮轴面轮缘处的压力大于轮毂处的 
压力, 进而使气体受到了由出口指向进口和由轮缘 指向轮毂的压力作用, $15 \%$ 进口含气率下叶轮流道 中间 S2 流面的轴面压力分布如图 9 所示。以上两点 共同造成了叶轮流道内的气体在出口轮敹吸力面附 近的聚集。因此, 对该类洜进行优化设计时, 可通 过改变出口面积、改变径向尺寸、增加泵的级数等 措施控制和调整逆压梯度, 进而减弱气体在流道内 的聚集程度。

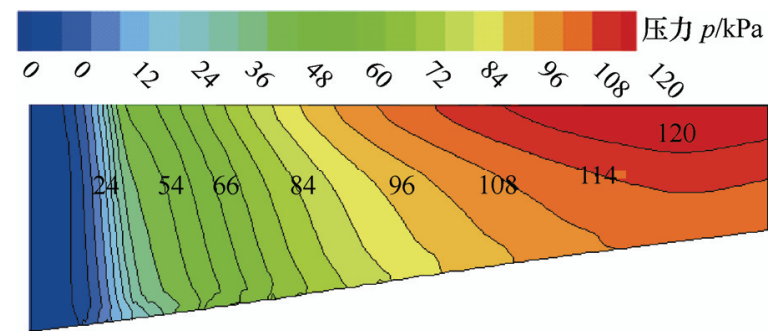

图 $915 \%$ 进口含气率下叶轮中间 S2 流面轴面压力分布

与单相流输送相比, 叶片式气液混输百在输送 过程中往往伴有相的分离和混合等复杂现象, 因此, 洜内流动比较复杂。图 10 是不同进口含气率下叶轮 流道内的湍动能分布。由图 10 可知, 随着进口含气 率的增加, 叶轮轮毂气体聚集区的湍动能逐渐增大; $9 \% 、 15 \%$ 和 $21 \%$ 进口含气率下叶轮流道内的最大湍 动能分别是 $3 \%$ 进口含气率下的 1.07 倍、 1.53 倍和 1.83 倍。说明随着进口含气率的增加, 气液相互作 用增强, 叶轮内流场更加紊乱, 这也是随着进口含 气率的增加, 泵扬程和效率逐渐降低的原因。

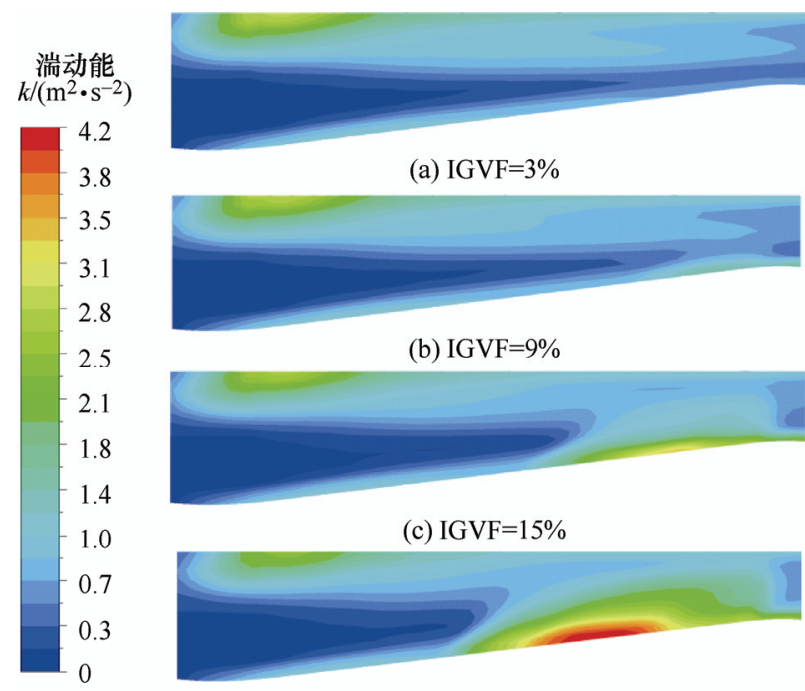

(d) IGVF=21\%

图 10 不同进口含气率下叶轮内流体湍动能分布

\section{3 进口含气率对导叶流道内流场特性的影响}

图 11 是不同进口含气率 $(\mathrm{IGVF}=3 \%, 9 \%, 15 \%$, $21 \%$ )下导叶进口到出口轴面的平均含气率分布(面 积平均)。由图 11 可知, 不同进口含气率下导叶流 道内气相分布规律相似: 均在轮毂处聚集, 且沿着

流动方向, 轮毂处的气体逐渐向主流区扩散。这是 因为在叶轮流道出口轮毂处聚集的气体，由于惯性 作用, 会继续向导叶流道内流动, 同时, 导叶流道 内离心力效应消失, 由于气相的密度小于液相的密 度，进而使导叶流道内的气体向下游移动的同时向 主流区扩散。
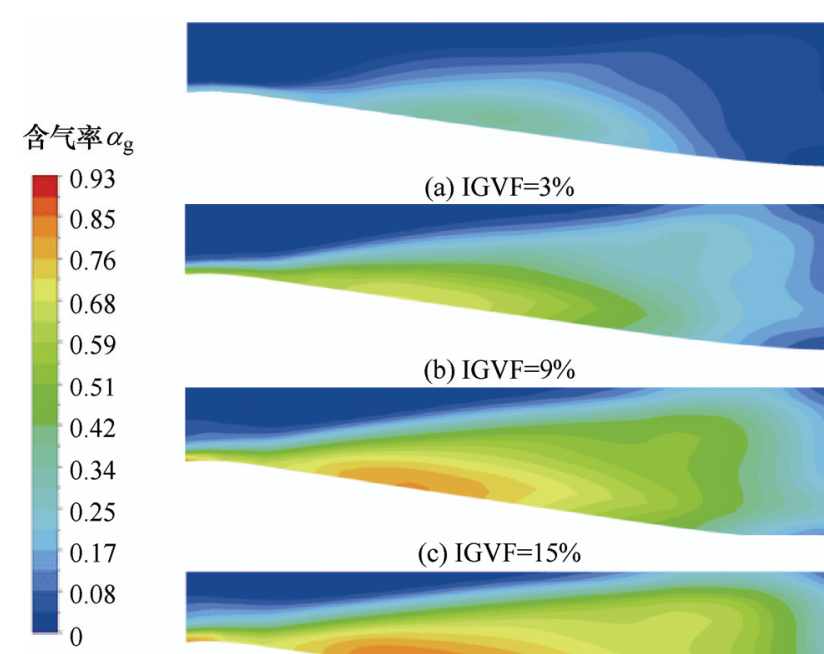

(a) $\mathrm{IGVF}=3 \%$

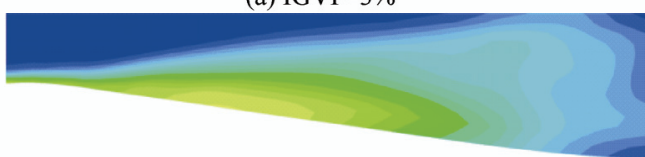

(b) $\mathrm{IGVF}=9 \%$

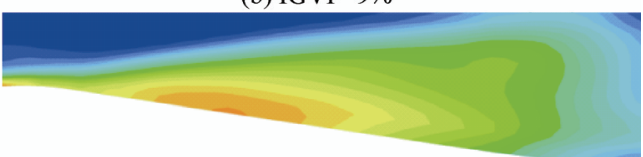

(c) $\mathrm{IGVF}=15 \%$

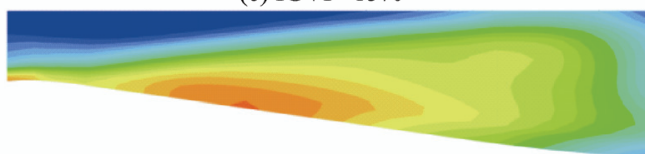

(d) IGVF $=21 \%$

图 11 不同进口含气率下导叶流道轴面含气率分布

图 12 是 $15 \%$ 进口含气率下导叶流道中间 S2 流 面的气体流线分布。由图 12 可知, 气体在导叶轮毂 处形成了明显的旋浴, 且从导叶进口到出口, 形成 旋浴的范围逐渐扩大, 这是因为导叶是渐扩形流道, 流体在导叶流道内的速度沿着流动方向逐渐减小, 且受到导叶流道中逆压梯度的影响, 进而使流体在 轮珲处发生了流动分离，形成了旋浴。

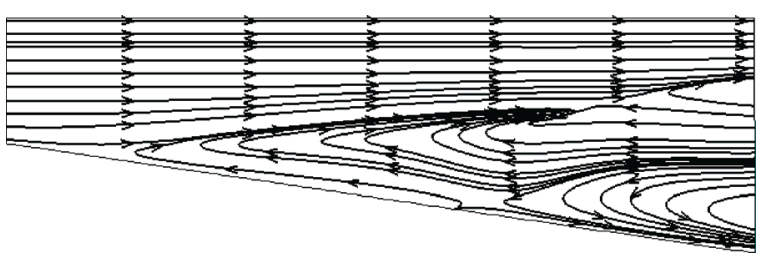

图 12 导叶流道中间 $\mathrm{S} 2$ 流面的气体流线分布 $(\mathrm{IGVF}=15 \%)$

图 13 是 $15 \%$ 进口含气率下导叶流道轴面气液 两相速度差分布。由图 13 可知, 导叶进口附近出现 了局部气液两相速度差较大的区域, 这是因为旋转 叶轮与静止导叶之间的动静干涉作用, 使动静干涉 区的流动状态较紊乱, 进而造成了该区域气液两相 速度差的增大; 而在导叶轮毂处的气液两相速度差 则较小, 结合图 11、12 分析可知, 该区域与导叶流 道轮毂处气体聚集区和旋涡形成区的位置相同, 说 明了导叶流道轮䟝处聚集的气体使该区域发生了气 液流动分离, 形成了旋涡, 并减小了该处的气液两 相速度差。 


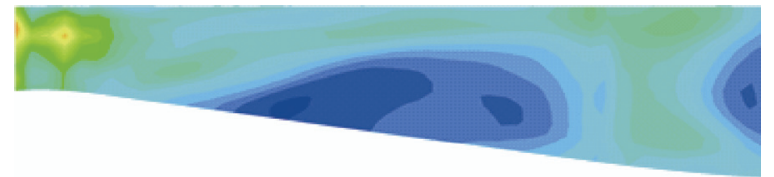

速度差 $\Delta U /\left(\mathrm{m} \cdot \mathrm{s}^{-1}\right)$

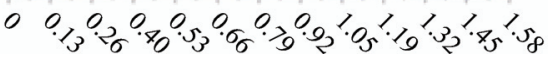

图 13 导叶流道内气液两相速度差分布 $(\mathrm{IGVF}=15 \%)$

\section{4 不同流量下叶轮和导叶流道内流场特性}

图 14 是当进口含气率为 $9 \%$ 时, 不同流量 $\left(0.75 Q_{\mathrm{d}}, Q_{\mathrm{d}}, 1.25 Q_{\mathrm{d}}\right)$ 下叶轮和导叶流道轴面的平均 含气率分布。由图 14 可知, 不同流量下叶轮和导叶 流道内的气体分布规律相似: 叶轮流道内的气体主 要在出口轮勃处聚集, 导叶流道轮教处聚集的气体 沿流动方向逐渐向主流区扩散; 同时, 叶轮和导叶 流道内, 气体聚集区均出现了旋浴及流动分离现象。

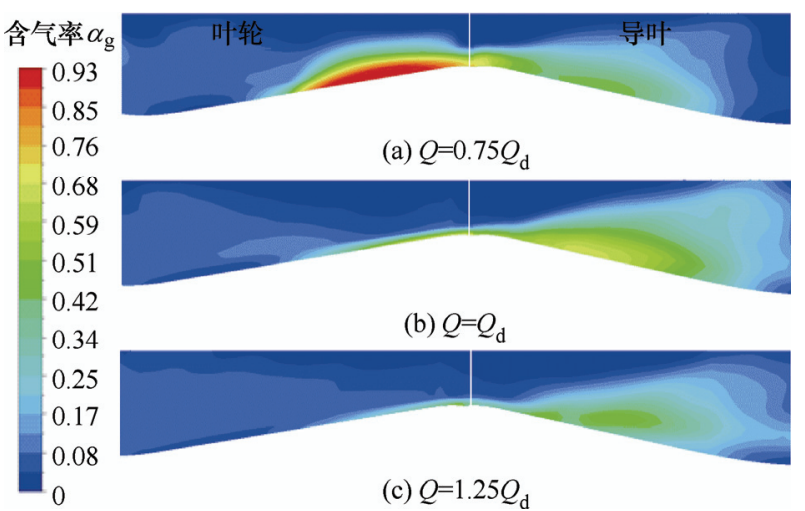

图 14 不同流量下叶轮和导叶流道轴面含气率分布 $(\mathrm{IGVF}=9 \%)$

随着流量的增加, 叶轮流道内气体的聚集程度 逐渐减小, 这是因为小流量 $\left(0.75 Q_{\mathrm{d}}\right)$ 下, 叶轮流道 气体聚集区出现了旋浴及流动分离现象, 如图 $15 \mathrm{a}$ 所示, 而随着流量的增加, 由于叶轮的做功作用, 液体的夹带能力增强, 该处的旋涡及气体聚集现象 消失, 如图 15b、15c 所示。由图 14 还可知, $Q=Q_{\mathrm{d}}$ 时, 导叶流道内气体的聚集程度最大, $1.25 Q_{\mathrm{d}}$ 次之, $0.75 Q_{\mathrm{d}}$ 最小。这与导叶流道内流体的流动状态密切

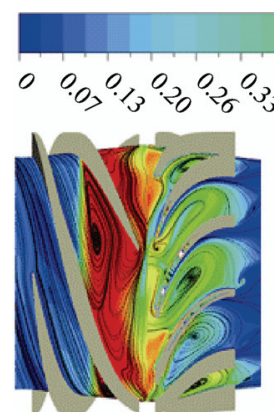

(a) $Q=0.75 Q_{\mathrm{d}}$ 含气率 $\alpha_{\mathrm{g}}$

图 15 叶轮和导叶流道轮毂处含气率和气体流线分布 $(\mathrm{IGVF}=9 \%)$
相关, 当 $Q=0.75 Q_{\mathrm{d}}$ 时, 气体在叶轮流道内聚集明显, 过流断面面积减小，从而增大了进入导叶流道内的 液体速度及其夹带能力, 因此该工况下气体在导叶 流道内的聚集程度相对较小; 而对于 $Q=1.25 Q_{\mathrm{d}}$ 时, 由于流体具有较大的动能, 从而使导叶流道内的旋 浴减小, 气液流动分离现象减弱, 进而使气体的聚 集程度较小。

\section{4 结论}

通过数值计算, 在不同进口含气率和不同流量 下对一气液两相混输百叶轮和导叶流道内的含气率 分布及内流场特性进行了探讨, 进一步了解了叶片 式混输泵内的气液两相流动规律。所得结论如下。

(1) 不同进口含气率下叶轮流道内的气体主要 在轮毂吸力面附近聚集且随着进口含气率的增加, 气体在该处的聚集程度增强, 分布的不均匀度增加, 流场更加紊乱。9 \% 、15\%和 21\%进口含气率下叶轮 流道内的最大湍动能分别是 $3 \%$ 进口含气率下的 1.07 倍、 1.53 倍和 1.83 倍。

(2) 不同进口含气率下导叶流道内气相分布规 律相似: 均在轮觳处聚集, 且从导叶流道进口到出 口, 轮毂处的气体逐渐向主流区扩散, 在轮毂处形 成的气体旋涡范围逐渐扩大。

(3) $9 \%$ 进口含气率下, 叶轮流道内气体的聚集 程度随着流量的增加逐渐减小, 而导叶流道内的气 体在设计流量 $\left(Q_{\mathrm{d}}\right)$ 时聚集程度最大, 大流量 $\left(1.25 Q_{\mathrm{d}}\right)$ 次之, 小流量 $\left(0.75 Q_{\mathrm{d}}\right)$ 最小。

\section{参 考 文 献}

[1] 张金亚, 蔡淑杰, 朱宏武. 叶片式混输百入口段气液两 相流场可视化试验 [J]. 机械工程学报，2015，51(18): 184-190.

ZHANG Jinya, CAI Shujie, ZHU Hongwu.Visualization test for flow field of gas-liquid two-phase in the entranceof rotodynamicmultiphase pump $[\mathrm{J}]$. Journal of Mechanical Engineering，2015， 51(18): 184-190.

[2] 王福军, 唐学林, 陈金金, 等. 泵站内部流动分析方法研 究进展 $[\mathrm{J}]$. 水利学报, 2018，49(1): 47-71.

WANG Fujun, TANG Xuelin, CHEN Xin, et al. A review on flow analysis method for pumping stations[J]. Journal of Hydraulic Engineering, 2018，49(1): 47-71.

[3] OMRANIA, FRANCHEK M, EBRAHIMI B. Lowdimensional modeling of a pumping unit to cope with multiphase[J]. Journal of Dynamic Systems Measurement \& Control, 2017, 139(4): 1-11.

[4] SUN J, KIM J, CHOI Y. A study on numerical 
optimization and performance verification of multi-phase pump for offshore plant $[\mathrm{J}]$. Proceedings of the Institution of Mechanical Engineers Part A: Journal of Power and Energy, 2017, 231(5): 382-397.

[5] KIM J H, LEE H C, KIM J H. Improvement of hydrodynamic performance of a multiphase pump using design of experiment techniques[J]. Journal of Fluids Engineering, 2015, 137(8): 1-15.

[6] CAO Shuliang, PENG Guoyi, YU Zhiyi. Hydrodynamic design of rotodynamic pump impeller for multiphase pumping by combined approach of inverse design and CFD analysis[J]. Journal of Fluids Engineering, 2005, 127(2): 330-338.

[7] MINEMURA K, MURAKAMI M. A theoretical study on air bubble motion in a centrifugal pump impeller[J]. Journal of Fluids Engineering, 1980, 102(4): 446-453.

[8] MINEMURA K, MURAKAMI M. Three-dimensional calculation of air-water twophase flow in centrifugal pump impeller based on a bubbly flow model[J]. Journal of Fluids Engineering, 1993, 115(4): 766-771.

[9] MINEMURA K, MURAKAMI M. Effects of entrained air on the performance of a centrifugal pumps: 2nd report, effects of number of blades [J]. Bulletin of the Japan Socitety of Mechanical Engineering, 1974, 40(330): 459-470.

[10] ZHANG Jinya, CAI Shujie, ZHU Hongwu. Experimental investigation of the flow at the entrance of arotodynamic multiphase pump by visualization[J]. Journal of Petroleum Science and Engineering, 2015，126: 254-261.

[11] CARIDAD J, ASUAJE M, KENYERY F. Characterization of a centrifugal pump impeller under two-phase flow conditions[J]. Journal of Petroleum Science and Engineering, 2008, 63(1): 18-22.

[12] YAN Di, KOVACEVIC A, TANGQiang, et al. Numerical modelling of twin-screw pumps based on computational fluid dynamics[J]. Proceedings of the Institution of Mechanical Engineers, Part C: Journal of Mechanical Engineering Science, 2017, 231(24): 4617-4634.

[13] ZHANG Wenwu, YU Zhiyi, ZHU Baoshan. Numerical study of pressure fluctuation in a gas-liquid two-phase mixed-flow pump[J]. Energies, 2017, 10(5): 1-14.

[14] 余志毅, 刘影. 叶片式混输葲气液两相非定常流动特性 分析[J]. 农业机械学报，2013，44(5): 66-69.

YU Zhiyi, LIU Ying. Characteristic analysis of unsteady gas-liquid two-phase flow in a multiphase rotodynamicpump $[\mathrm{J}]$. Transactions of the Chinese Society of Agricultural Machinery, 2013, 44(5): 66-69.

[15] LEE D, BEST F R, MCGRAW N. Microgravity two-phase flow regime modeling[C]// Proceedings of the Nuclear Society Winter Meeting, Los Angeles, CA, USA, 15 November, 1987.

[16] BENHMIDENE A, CHAOUCHI B, BOUROUIS M. Numerical prediction of flow patterns in bubble pumps[J]. Journal of Fluids Engineering, 2011, 133(3): 1-8.

[17] SITU R, HIBIKI T, BROWN RJ. Flow regime transition criteria for two-phase flow at reduced gravity conditions[J]. International Journal of Multiphase Flow, 2011, 37(9): 1165-1177.

[18] SUH J W, KIM J W, CHOI Y S, et al. Development of numerical Eulerian-Eulerian models for simulating multiphase pumps[J]. Journal of Petroleum Science and Engineering, 2018, 162: 588-601.

[19] ZHU Jianjun, ZHANG Hongquan. Mechanistic modeling and numerical simulation of in-situ gas void fraction inside ESP impeller [J]. Journal of Natural Gas Science and Engineering, 2016, 36: 144-154.

[20] ZHANG Wenwu, YU Zhiyi, ZAHID M N, et al. Study of the gas distribution in a multiphase rotodynamicpump based on interphase force analysis[J]. Energies, 2018, 11(5): 1-16.

[21] TAN Lei, ZHU Baoshan, CAO Shuliang. Numerical simulation of unsteady cavitation flow in a centrifugal pump at off-design conditions[J]. Proceedings of the Institution of Mechanical Engineers, Part C: Journal of Mechanical Engineering Science, 2013 , 228(11) : 1994-2006

[22] TAN Lei, ZHU Baoshan, CAO Shuliang. Cavitation flow simulation for a centrifugal pump at a low flow rate[J]. Chinese Science Bulletin, 2013， 58 (8): 949-952.

[23] PEN Ji, YIN Tingyun, YUAN Shouqi, et al. Cavitation optimization for a centrifugal pump impellerby using orthogonal design of experiment[J]. Chinese Journal of Mechanical Engineering, 2017, 30(1): 103-109.

[24] 张文武, 余志毅, 祝宝山, 等. 叶顶间隙对低比转速混 流洜性能及内部流场影响的数值研究 $[\mathrm{J}]$. 机械工程学 报，2017，53(22): 182-189.

ZHANG Wenwu, YU Zhiyi, ZHU Baoshan, et al. Study of tip clearance effects on performances and flow field of a Lowspecific speed mixed-flow pump[J]. Journal of Mechanical Engineering, 2017, 53(22): 182-189.

作者简介: 张文武, 男, 1989 年出生, 博士研究生。主要从事流体机械 流动理论及数值模拟方法的研究。

E-mail: wwzhang@bit.edu.cn

余志毅(通信作者), 男, 1976 年出生, 博士, 副教授。主要从事流体机 械流动理论及数值模拟方法的研究。

E-mail: yuzhiyi@bit.edu.cn 